\title{
Nlrp3-dependent IL-1ß inhibits CD103+ dendritic cell differentiation in the gut
}

\author{
Rachel Mak'Anyengo, ${ }^{1}$ Peter Duewell,, Cornelia Reichl, ${ }^{1}$ Christine Hörth, ${ }^{1}$ Hans-Anton Lehr, ${ }^{2}$ \\ Sandra Fischer, ${ }^{3}$ Thomas Clavel, ${ }^{3,4}$ Gerald Denk, ${ }^{5}$ Simon Hohenester, ${ }^{5}$ Sebastian Kobold, ${ }^{1}$ \\ Stefan Endres, ${ }^{1}$ Max Schnurr, ${ }^{1}$ and Christian Bauer ${ }^{6}$ \\ ${ }^{1}$ Center of Integrated Protein Science Munich (CIPS-M) and Division of Clinical Pharmacology, Medizinische Klinik und \\ Poliklinik IV, Klinikum der Universität München, Munich, Germany. ${ }^{2}$ Institute of Pathology, Medizin Campus Bodensee, \\ Friedrichshafen, Germany. ${ }^{3}$ Core Facility Microbiome, ZIEL-Institute for Food and Health, Technische Universität München, \\ Freising-Weihenstephan, Germany. ${ }^{4}$ Institute of Medical Microbiology, RWTH University Hospital, Aachen, Germany. \\ ${ }^{5}$ Medizinische Klinik und Poliklinik II, Klinikum der Universität München, Munich, Germany. ${ }^{6}$ Division of Gastroenterology, \\ Endocrinology, Infectiology and Metabolism, University Hospital Giessen and Marburg, Campus Marburg, Philipps \\ University Marburg, Marburg, Germany.
}

Inflammatory bowel disease (IBD) is associated with enhanced levels of the IL-1 family cytokines IL-1 $\beta$ and IL-18, which are activated by the NIrp3 inflammasome. Here, we investigated the role of inflammasome-driven cytokine release on T cell polarization and DC differentiation in steady state and T cell transfer colitis. In vitro and in vivo data showed that IL-1ß induces Th17 polarization and increases CM-CSF production by T cells. Reduced IL-1 $\beta$ levels in $\mathrm{NIrp3}^{-1 /}$ mice correlated with enhanced FLT3L levels and increased frequency of tolerogenic CD103+ DC. In the T cell transfer colitis model, NIrp3 deficiency resulted in lower IL-1 $1 \beta$ levels, reduced Th17 immunity, and less severe colitis. Unaltered IL-18 levels in both mouse strains pointed toward NIrp3-independent processing. Importantly, cohousing revealed that the gut microbiome had no impact on the observed $\mathrm{NIrp3}^{-/-}$phenotype. This study demonstrates that NLRP3 acts as a molecular switch of intestinal homeostasis by shifting local immune cells toward an inflammatory phenotype via IL-1及.

Authorship note: RM and PD contributed equally to this work.

Conflict of interest: The authors have declared that no conflict of interest exists.

Submitted: July 17, 2017

Accepted: February 7, 2018

Published: March 8, 2018

Reference information:

JCI Insight. 2018;3(5):e96322. https:// doi.org/10.1172/jci.insight.96322.

\section{Introduction}

The gastrointestinal tract comprises the body's largest interface toward daily environmental factors that influence inflammatory pathways, whereas genetic alterations provide a source of inherent mutations, associated with intestinal inflammation and the predisposition to inflammatory bowel disease (IBD) $(1,2)$. The intracellular NOD-like receptor (NLR) NOD2/IBD1 was the first identified gene associated with a risk of developing Crohn's disease (3, 4). NACHT, LRR, and PYD domains-containing protein 3 (NALP3) is one of the best-characterized NLRs, able to oligomerize with the adaptor apoptosis-associated speck-like protein containing a CARD (ASC) and caspase-1 to form a multiprotein platform, termed the inflammasome (5). Unlike other inflammasomes, the ligand for NLRP3 remains elusive. Danger- and pathogen-associated molecular patterns, such as crystals or aggregated proteins, bacterial toxins, and ROS have been shown to activate inflammasome signaling (6). Upon assembly, caspase- 1 is activated to process IL- $1 \beta$ and IL-18 into their active and secreted forms. Mutations of the NLRP3 gene have been linked to rare inherited autoinflammatory diseases, summarized as cryopyrin-associated periodic syndrome (CAPS) (7). NLRP3 inflammasome activation is tightly regulated. It requires an initial priming step for the transcription of NLRP3, pro-IL-1 $\beta$ and pro-IL-18 and a second activation step leading to the secretion of the bioactive cytokines (6). Both IL-1 $\beta$ and IL-18 share common downstream signaling features, and the association with the pathogenesis of IBD leads back to the early 1990s (8-10).

In clinical studies, IL-1 $\beta$ levels have been reported to correlate with disease activity (11) and to act in concert with other proinflammatory cytokines to induce Th17 cells, which are key mediators of both Crohn's disease and ulcerative colitis $(12,13)$. The role of IL-18 in IBD is a matter of ongoing debate (14-16). Although elevated IL-18 levels are observed in IBD patients and in animal models, both protective and deleterious effects of IL-18 signaling have been reported $(17,18)$. One explanation is that intestinal IL-1 $\beta$ is mostly produced by myeloid cells, whereas IL-18 is constitutively expressed in epithelial cells and seems to regulate mucosal homeostasis (18). 
The role of Nlrp3 in IBD has been mostly investigated using the model of dextran sulfate sodiuminduced (DSS-induced) colitis. Our group has described a protective effect of NLRP3 deficiency in acute DSS colitis (19). The role of NLRP3- and IL-1 $\beta$-mediated colonic inflammation has recently been confirmed by a study on Nlrp3 regulation by miR-223 (20). We hypothesized that DSS compromises gut barrier integrity and allows priming of NLRP3 by bacterial components, with subsequent initiation of caspase-1mediated IL-1 $\beta$ release by myeloid cells within the lamina propria (LP). Similar results have been observed in caspase-1-deficient mice or by administration of soluble IL-1Ra antibody, which both led to profound amelioration of DSS-induced colitis (21). In addition, our group has shown that pralnacasan, a small molecule caspase-1 inhibitor, significantly reduced severity of DSS colitis $(19,22)$. Other groups have reported

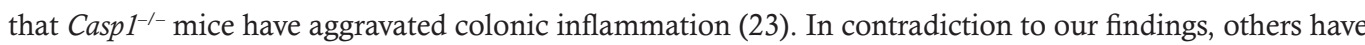

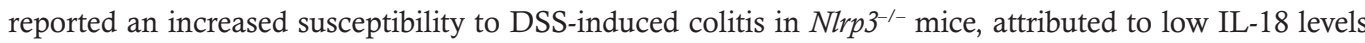
and defective epithelial repair $(14,24)$. In line with this, $I l-18^{-1-}$ and $I l-18 R^{-1-}$ mice were shown to develop more severe DSS-induced colitis compared with WT mice (25). However, reduced disease severity of DSS-induced colitis was shown by other studies after treatment with anti-IL-18 antibody or IL-18-binding protein $(26,27)$. Interestingly, the study by Neudecker et al. found that IL-18 levels were unaltered by Nlrp 3 expression (20). Recently, it was demonstrated that transfer of $I l-18 R^{-/-} \mathrm{T}$ cells did not suppress $\mathrm{T}$ cell transfer colitis, although IL-18R signaling was required for generation and maintenance of optimal Th1 T cell responses, indicating a possible dichotomal role of IL-18 in colitis (28).

Thus, a large body of evidence reveals a role for the NLRP3/caspase-1/IL-1 $\beta$ axis in IBD; however, experimental protocols and the genetic background of mice may affect results $(14,24,29)$. Considering the peculiarities of biochemical inhibitor studies, including off-target effects versus genetic approaches with altered microbiome, most disease models currently available are susceptible to microbial diversity. In line with this, we have previously reported that cohousing led to comparable susceptibility to colitis-inducing agents in $\mathrm{Nlrp3}^{-1-}$ and WT mice, and that treatment with antibiotics significantly reduced disease severity (30).

Intestinal DC comprise multifaceted cell populations with antigen presenting activity, effector $\mathrm{T}$ cell stimulation, and induction of Treg differentiation, depending on the local stimulus (31-34). Amongst these, local CX3CR $1^{+} \mathrm{CD} 11 \mathrm{~b}^{+}$monocyte-derived DC directly interact with the intestinal lumen while, migratory $\mathrm{CD}_{103}{ }^{+} \mathrm{CD} 11 \mathrm{c}^{+} \mathrm{MHC}-\mathrm{II}^{+} \mathrm{DC}$ mediate T cell homing and differentiation $(35,36)$. Both DC subsets have different progenitors, with $\mathrm{CX} 3 \mathrm{CR} 1^{+} \mathrm{CD} 11 \mathrm{~b}^{+} \mathrm{DC}$ originating from macrophage precursors and $\mathrm{CD} 103^{+} \mathrm{DC}$ from DC progenitor cells. The balance of $\mathrm{CD}_{103^{+}}$and $\mathrm{CD} 103^{-} \mathrm{DC}$ preserves gut homeostasis and is tightly regulated by cytokines such as FMS-like tyrosine kinase 3 ligand (FLT3L) and GM-CSF. FLT3L is required for $\mathrm{CD}_{103}{ }^{+} \mathrm{DC}$, whereas GM-CSF seems to influence $\mathrm{CD} 11 \mathrm{~b}^{+}$expression $(37,38)$. Intestinal CD $103^{+} \mathrm{DC}$ are considered tolerogenic and promote Treg function $(32,38)$.

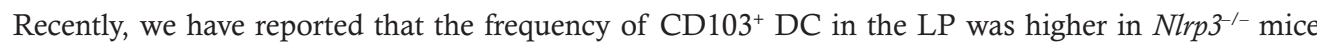
compared with WT mice at steady state (30). In the present study, we investigated the molecular basis for this observation and elucidated how Nlrp3 links innate to adaptive immune responses in intestinal DC using a $\mathrm{T}$ cell transfer colitis model.

\section{Results}

NLRP3 inflammasome limits FLT3L-mediated differentiation of tolerogenic CD103+ DC in vitro and in vivo. We recently reported an increased frequency of $\mathrm{CD} 103^{+} \mathrm{DC}$ in the LP of $\mathrm{Nlrp} 3^{3^{--}}$mice. As CD $103^{+} \mathrm{DC}$ are considered to exert tolerogenic functions, we hypothesized that this finding might explain reduced colitis severity in $\mathrm{Nlrp3}^{-/-}$mice, as observed in DSS- and TNBS-induced colitis models $(19,30)$. To elucidate the relevance of our finding, we analyzed immune cells from LP, mesenteric lymph node, and spleen of WT and $\mathrm{Nlrp}^{3^{-1}}$ mice in steady state. The number of total CD11c $\mathrm{c}^{+} \mathrm{DC}$ was similar in both genotypes, but the

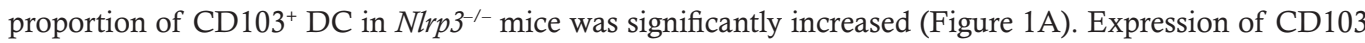
by DC requires the growth factor FLT3L $(37,38)$. For further characterization of the observed DC phenotype, we generated BM DC of WT and $\mathrm{Nlrp}^{-/-}$mice using the GM-CSF + IL-4 or the FLT3L protocol. FACS analysis confirmed high frequency of $\mathrm{CD} 103^{+}$cells within the FLT3L-differentiated WT and Nlrp ${ }^{-1-}$ DC, whereas the GM-CSF + IL-4 protocol resulted in low numbers of CD103 ${ }^{+}$cells. Within FLT3L cultures, $\mathrm{Nlrp}^{3^{-/}}$DC expressed significantly higher CD103 levels when compared with DC from WT mice, pointing out a role for NLRP3 in controlling CD103+ DC differentiation (Figure 1B).

Flt3L DC cultures were shown to produce $\mathrm{CD} 103^{+} \mathrm{DC}$ with reduced cytokine production and tolerogenic features (39). This led us to investigate the inflammatory potential of DC from WT and $N \mathrm{Irp}^{3^{-1-}}$ mice 
A WT $\square \mathrm{NIrp3}^{-1}$
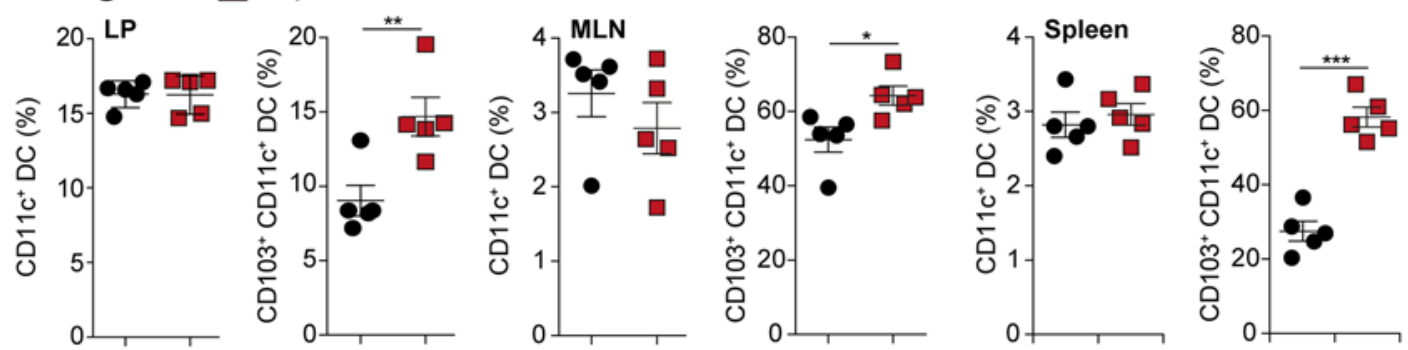

B
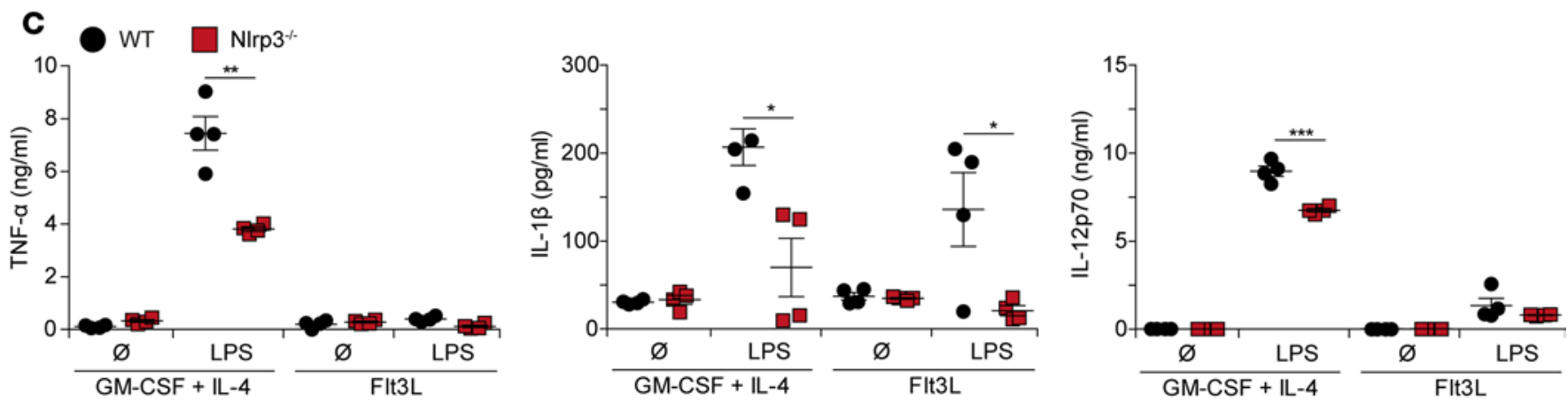

D
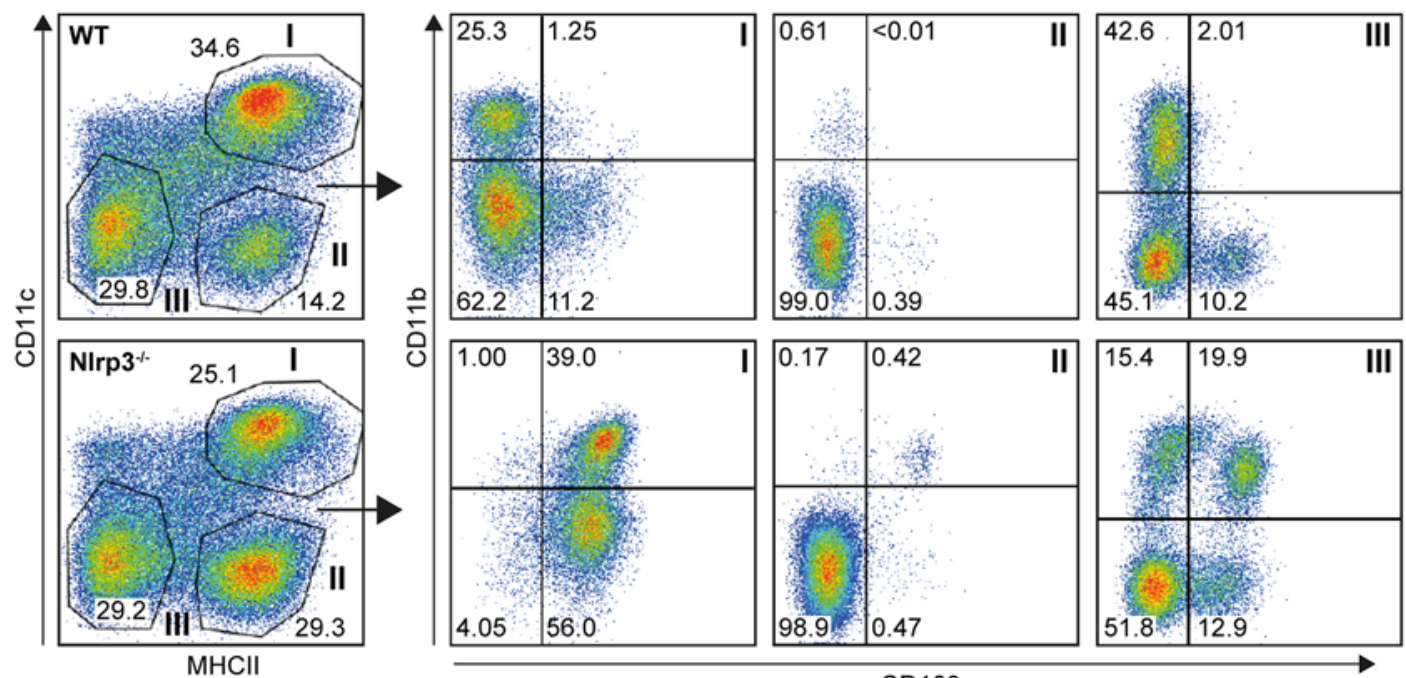

CD103

E

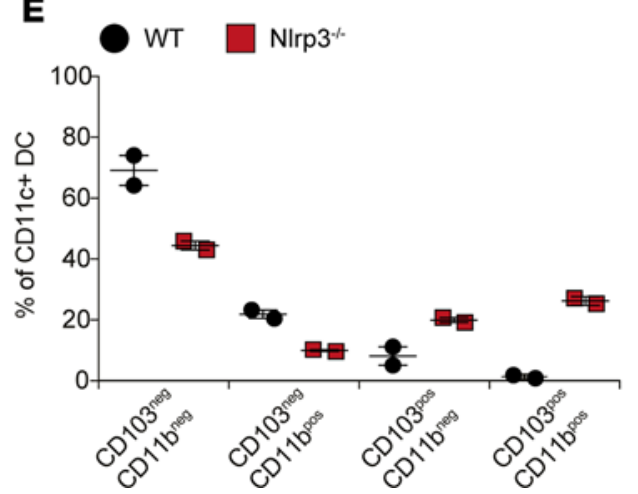

$\mathbf{F}$
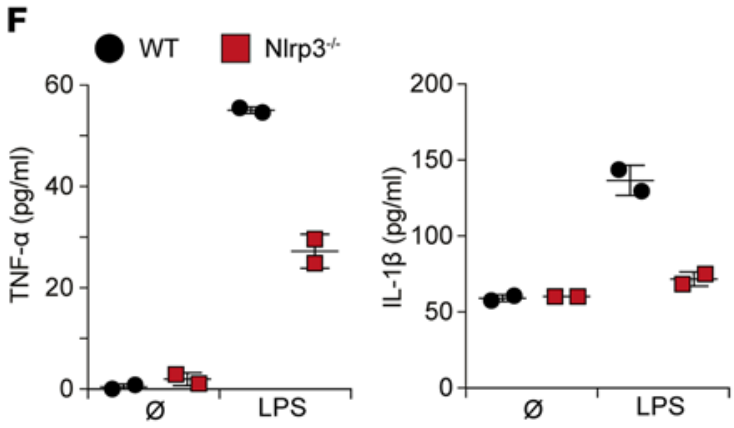

Figure 1. NLRP3 deficiency favors development of tolerogenic $\mathrm{CD}_{103^{+}} \mathrm{DC}$ in a FLT3L-dependent manner. (A) Frequency of total DC (CD11C $\left.{ }^{+} \mathrm{MHCII^{+ }}\right)$ and percentage of $\mathrm{CD}_{103^{+}} \mathrm{DC}$ in lamina propria (LP), mesenteric lymph node (MLN), and spleen of WT and N/rp3 $3^{-/-}$mice under steady state conditions. (B) Development of $\mathrm{CD}_{103^{+}} \mathrm{DC}$ from BM precursors of WT and $\mathrm{N}_{\mathrm{rpp}} 3^{-/-}$mice using either the GM-CSF + IL-4 or FLT3L protocol. (C) Cytokine secretion of BM-derived DC from WT and N/rp3 $3^{-1-}$ mice stimulated overnight with LPS. Levels of TNF- $\alpha$, IL-1 $\beta$, and IL-12(p70) were measured in supernatants 
with ELISA. (D-F) WT and N/rp3 $3^{-1-}$ mice were implanted with FLT3L-producing B16 tumor cells. (D and E) Expression profile of whole splenic subpopulations (D) and MACS-sorted CD11 ${ }^{+}$DC populations (E) were analyzed by FACS. (F) CD11c-enriched DC were stimulated with LPS overnight. Secretion of TNF- $\alpha$ and IL-1 $\beta$ into supernatant was determined by ELISA. Data are shown as mean \pm SEM; (A) WT $n=5, N / r p 3^{-/-}, n=5,1$ out of 3 experiments is shown; (B) WT, $n=4$, NIrp3 $^{-1-}, n=4,1$ out of 3 experiments shown; (C) WT $(n=4)$, Nlrp3-1- $(n=4)$, 1 out of 3 experiments shown; (D-F) WT ( $\left.n=2\right)$, Nlrp3 $^{-1-}(n=2), 1$ out of 2 experiments is shown. ${ }^{*} P<0.05,{ }^{* *} P<0.01,{ }^{* *} P<0.001$, as assessed by unpaired 2-tailed Student's $t$ test.

after stimulation with the TLR4 ligand LPS. Compared with FLT3L, GM-CSF-differentiated DC produced high levels of the proinflammatory cytokines TNF, IL-1 $\beta$, and IL-12p70. Compared with WT DC, cytokine production was significantly lower in $N l r p 3^{-1-}$ DC. In FLT3-differentiated DC, IL-1 $\beta$ secretion was almost absent in Nlrp $3^{--}$DC, in line with inflammasome-mediated IL-1 $\beta$ processing (Figure 1C). In conclusion, Nlrp3 deficiency favors development of $\mathrm{CD}_{103^{+}} \mathrm{DC}$ with reduced inflammatory capacity.

We next investigated the role of FLT3L-dependent DC expansion in vivo. We injected WT or Nlrp3-1mice with FLT3L-producing B16 melanoma cells and assessed the frequency of splenic CD103 ${ }^{+} \mathrm{DC}$ populations (Figure 1D). As expected, high FLT3L levels led to strong expansion of DC populations. Compared with WT mice, a massive shift from CD103- toward CD103+ DC, in both CD11b and CD11 b ${ }^{-}$DC populations, was observed in NLRP3-deficient mice (Figure 1, D and E). DC expanded in WT mice secreted higher levels of TNF- $\alpha$ and IL-1 $\beta$ than DC from Nlrp $3^{-1-}$ mice in response to LPS stimulation (Figure 1F). Together, these in vivo and in vitro findings indicate a decisive role for the NLRP3/IL-1 $\beta$ axis in controlling CD103 expression by DC.

NLRP3-dependent IL-1 $\beta$ produced by DC limits FLT3L but enhances GM-CSF and IL-17 secretion by T cells. Upon $\mathrm{DC}$ and $\mathrm{T}$ cell interaction, $\mathrm{T}$ cells are polarized into an effector or regulatory phenotype with secretion of corresponding cytokines and growth factors. We hypothesized that NLRP3 in DC influences intestinal $\mathrm{T}$ cell phenotype and function. Hence, we analyzed cytokine secretion of naive CD4 $4^{+} \mathrm{OT}$-II T cells cocultured with splenic, OVA peptide-pulsed DC. T cells cocultured with $\mathrm{Nlrp}^{3^{--}}$DC produced significantly more FLT3L but lower levels of GM-CSF, as compared with WT DC. In addition, IL-17 levels were significantly reduced, whereas secretion of the Th1 cytokine IFN- $\gamma$ was similar for both DC genotypes (Figure 2A). These findings argue for an NLRP3-dependent role in DC balancing GM-CSF and FLT3L secretion, as well as Th17 polarization of $\mathrm{T}$ cells.

The NLRP3 inflammasome controls the activation of IL-1 $\beta$ and IL-18, which are both involved in $\mathrm{T}$ helper cell development (40). To investigate the differential contribution of IL-1 $\beta$ and IL-18 on T cell polarization, we stimulated CD4+ $\mathrm{T}$ cells with IL-1 $\beta$ or IL-18 in the presence of antibody-mediated CD3/ CD28 costimulation. The addition of IL-18 increased the production of FLT3L by activated T cells, whereas IL-1 $\beta$ enhanced GM-CSF production (Figure $2 \mathrm{~B}$ ). Moreover, only IL-1 $\beta$ induced the secretion of the Th17-associated cytokines IL-17 and IL-22, yet with similar levels of IFN- $\gamma$ for both IL-1 family cytokines

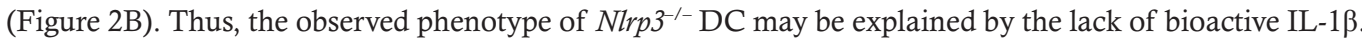

As our in vitro data indicated a link between NLRP3 and FLT3L, we investigated the in vivo levels of FLT3L and GM-CSF in colonic tissue and mesenteric lymph nodes of WT and $\mathrm{Nlrp}^{3^{-1}}$ mice under steady state conditions. Compared with WT mice, $\mathrm{Nlrp}^{-1-}$ mice exhibited significantly higher FLT3L tissue levels (Figure 2C). GM-CSF levels, however, were reduced but did not reach statistical significance at this point. As our results identified T cells as a source of both growth factors upon activation by DC and IL-1 family cytokines, we isolated $\mathrm{CD} 4^{+} \mathrm{T}$ cells from mesenteric lymph nodes and spleen and analyzed them for cytokine expression by quantitative PCR (qPCR). We observed significantly increased FLT3L mRNA expression in T cells of $\mathrm{Nlrp}^{---}$mice and higher expression of GM-CSF mRNA in WT mice (Figure 2D). Together, these data show that NLRP3-mediated IL-1 $\beta$ tips the FLT3L/GM-CSF balance in T cells toward GM-CSF, which prevents the development of tolerogenic $\mathrm{CD} 103^{+} \mathrm{DC}$ favoring Th17-associated pathology.

Enhanced frequency of $\mathrm{CD} 103^{+} \mathrm{DC}$ in Nlrp3-1- mice correlates with reduced Th17 immunity and protection from $T$ cell transfer colitis. Rag1 $1^{-/-}$mice lack functional $\mathrm{B}$ and $\mathrm{T}$ cells and are used to study the pathogenesis of colitis by adoptively transferred $\mathrm{T}$ cells. This model provides a platform for host-mediated education of naive $\mathrm{T}$ cells and their interaction with local DC. Naive CD4 $4^{+} \mathrm{T}$ cells were transferred into $\mathrm{Rag}^{1^{--}}$or $\mathrm{Nlrp}^{-{ }^{--}} \mathrm{Rag}^{1^{-1}}$ mice, and local DC as well as T cells were analyzed in different tissues after 4 weeks. Similar to our previous observations, the frequency of total CD11 $\mathrm{c}^{+} \mathrm{DC}$ in $\mathrm{LP}$ was comparable, but again, $\mathrm{CD} 103^{+} \mathrm{DC}$ were significantly increased in NLRP3-deficient Rag1 $1^{--}$mice (Figure 3A). Increased numbers of $\mathrm{CD} 103^{+} \mathrm{DC}$ correlated with significantly enhanced FLT3L levels and reduced GM-CSF mRNA expression in colon tissue of Nlrp3-1Rag $1^{-1-}$ hosts (Figure 3B). Colon tissue levels of the inflammatory cytokines IL-1 $\beta$, TNF, and IL-6 were also 
A
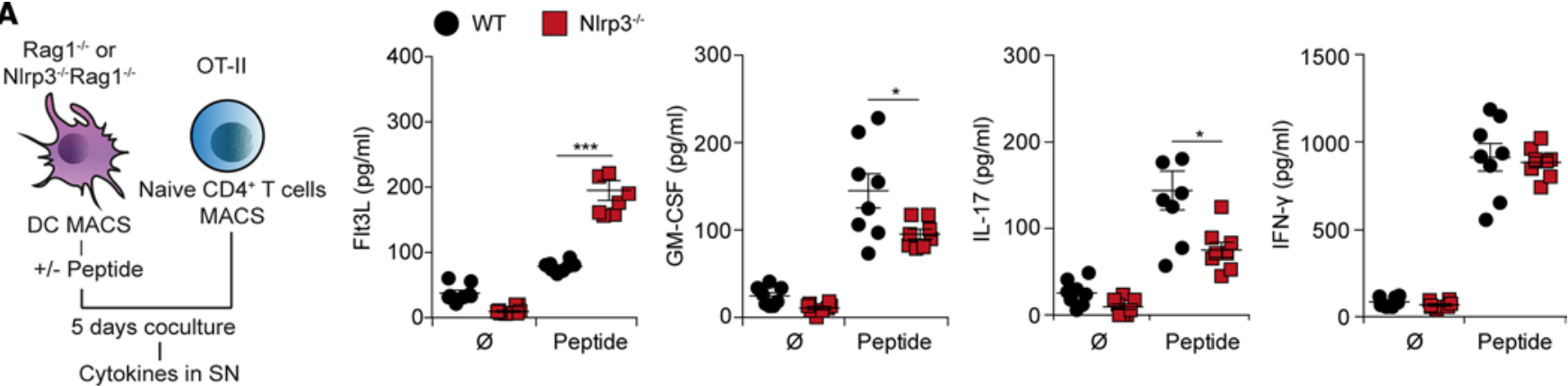

B
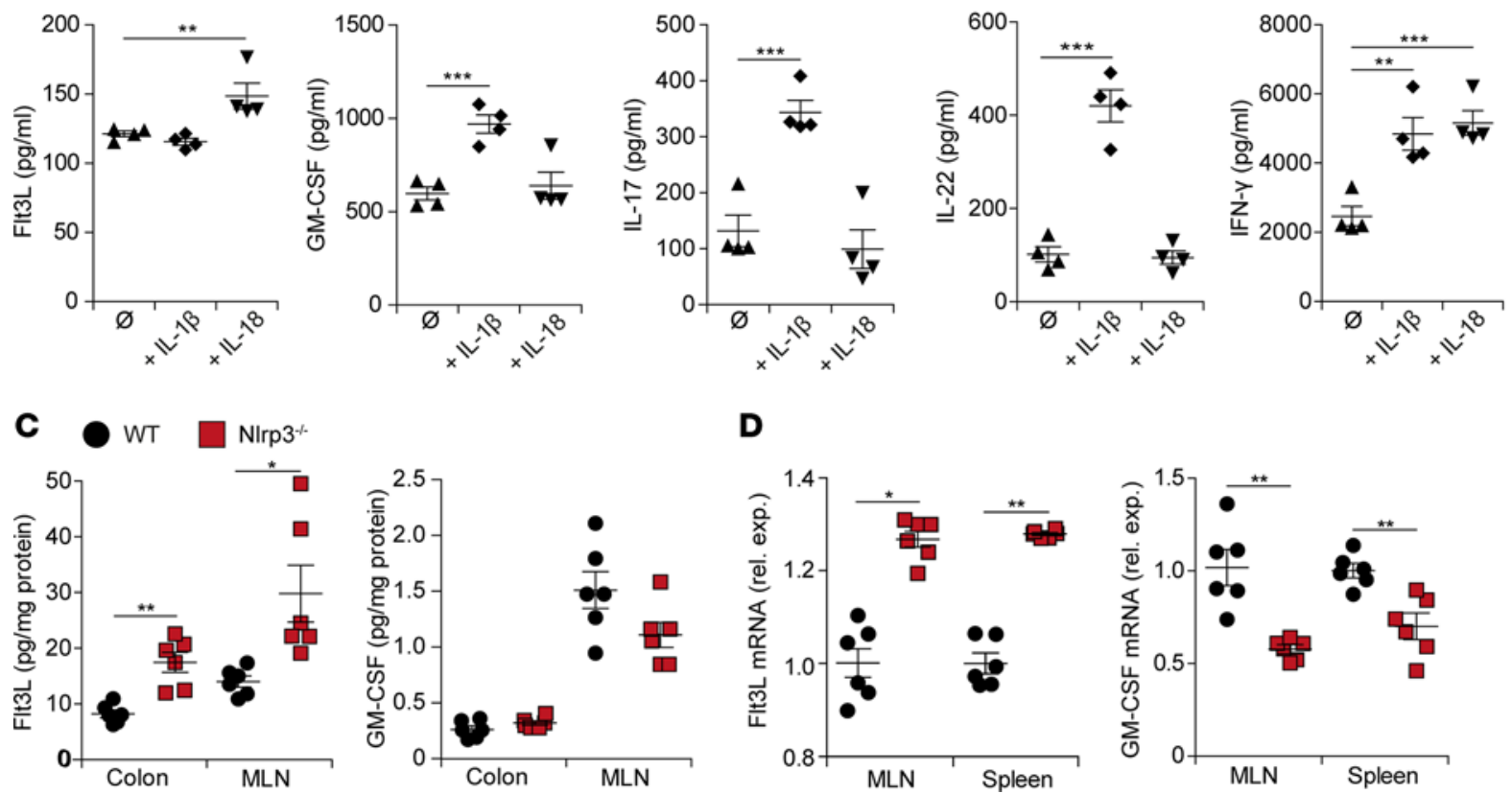

D
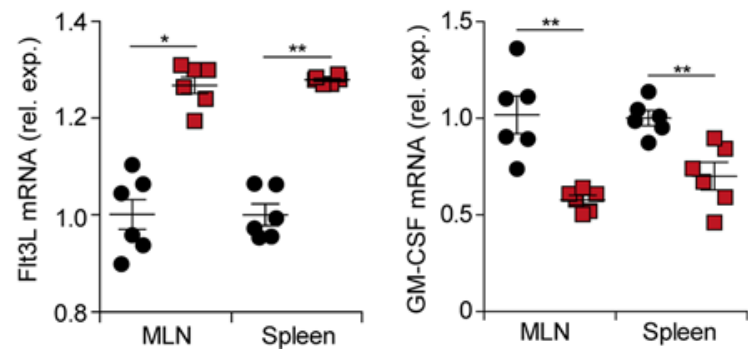

Figure 2. NIrp3-dependent IL-1 $\beta$ controls GM-CSF and FLT3L secretion by T cells and Th17 polarization. (A) MACS-enriched splenic DC from Rag1-1- and

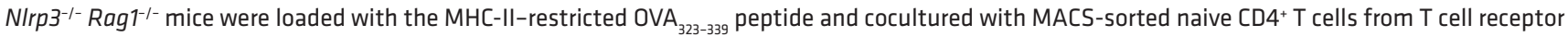
(TCR) transgenic OT-II mice for 5 days. FLT3L, GM-CSF, IFN- $\gamma$, and IL-17 levels in supernatants were measured by ELISA. (B) MACS-sorted splenic CD4+ T cells from WT mice were activated with anti-CD3 and anti-CD28 mAb in the absence or presence of IL-1 $1 \beta$ or IL-18 for 3 days. Secretion of FLT3L, GM-CSF, IL-17, IFN- $\gamma$, and IL-22 was measured by ELISA. (C) Colon and mesenteric lymph nodes from WT and NIrp3 ${ }^{-1-}$ mice at steady state were analyzed for FLT3L and GM-CSF production by ELISA. (D) CD4 ${ }^{+} T$ cells from mesenteric lymph nodes and spleen were FACS-sorted, and mRNA expression levels of FLT3L and GM-CSF were analyzed by qPCR. Data are shown as mean \pm SEM. (A) Pooled data from 3 independent experiments are shown; WT, $n=8 ; N / r p 3^{-1-}, n=8$.

(B) One out of 3 independent experiments is shown; WT, $n=4$; $\mathrm{NIrp3}^{-1-}, n=4$. (C and D) Pooled data from 3 independent experiments are shown; WT, $n=6$; NIrp3 $3^{-1-}, n=6 .{ }^{*} P<0.05,{ }^{* *} P<0.01,{ }^{* *} P<0.001$, as assessed by unpaired 2-tailed Student's $t$ test.

strongly reduced, whereas induction of IL-18 was comparable in both groups (Figure 3C). IL-17 and IL-22 secretion in colon tissue of $\mathrm{Nlrp}^{3^{-1}} \mathrm{Rag}^{-1-}$ hosts was strongly decreased, correlating with reduced frequency of IL-17-producing T cells (Figure 3D).

The key question was whether the observed increase in $\mathrm{CD}_{103^{+}} \mathrm{DC}$ and reduced $\mathrm{Th} 17$ immunity

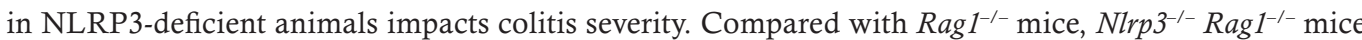
experienced reduced weight loss, diarrhoea, rectal bleeding, and tissue damage, as assessed by clinical scoring and histology (Figure 3, E-G). These findings demonstrate the importance of the NLRP3/ IL-1 $\beta$ axis for inducing Th17 immunity.

IL-18R signaling in T cells is protective in T cell-mediated colitis. Unaltered IL-18 colon levels in Rag1/-- and $N_{l r p 3^{-1}}$ Rag1 $^{-^{-1}}$ mice indicate an NLRP3-independent mechanism for IL-18 production (23, 41, 42). Given the potent proinflammatory function of IL-1 $\beta$ in the colitis model, we were interested in the role of IL-1R and IL-18R signaling in T cells. For this, we transferred WT, Illr1 $1^{-/-}$, or $I l 18 r 1^{-1-} \mathrm{CD} 4^{+} \mathrm{T}$ cells into $\mathrm{Rag}^{1^{--}}$mice and assessed disease progression. Clinical and histological scoring revealed that $I l 18 r 1^{-1-} \mathrm{T}$ cells 
A

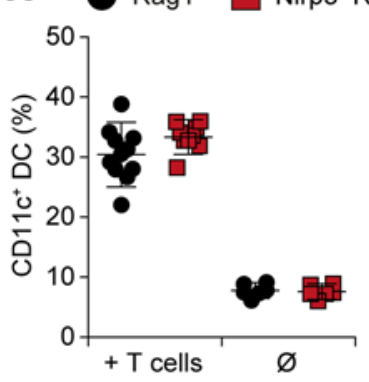

C

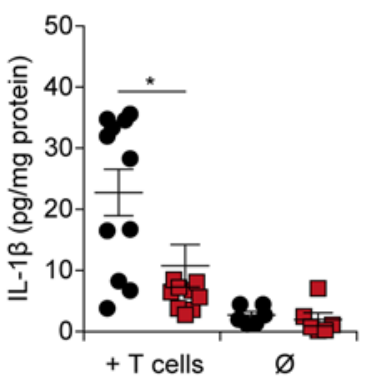

D

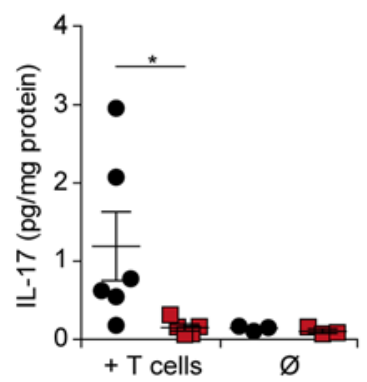

$\mathbf{F}$

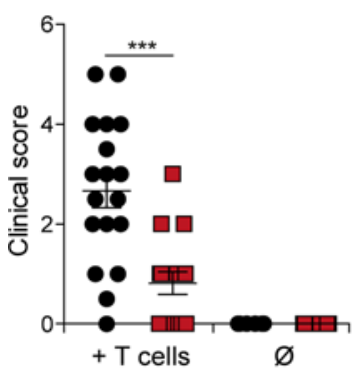

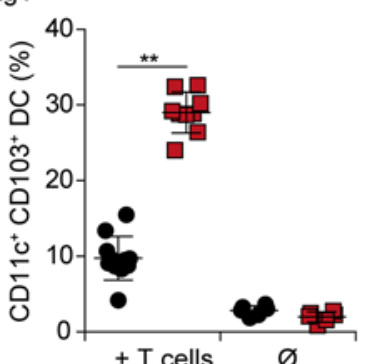
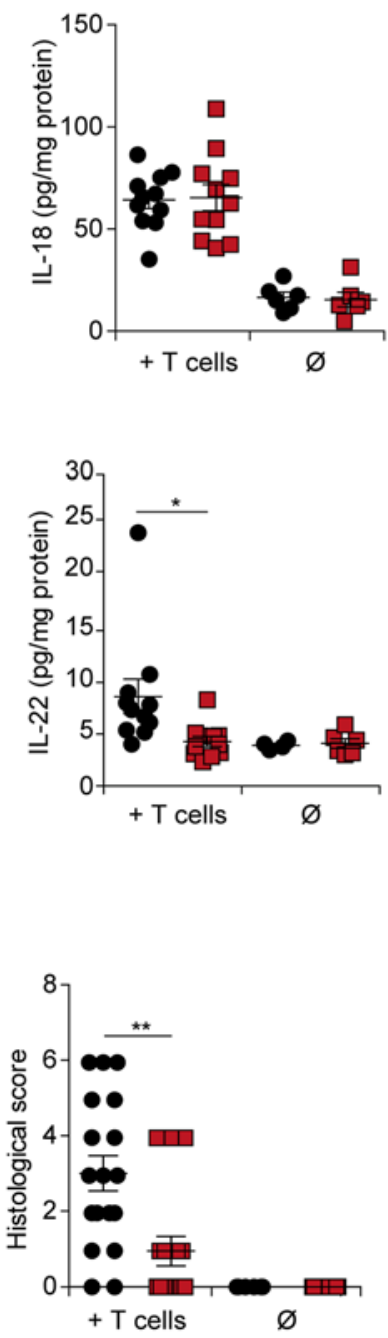
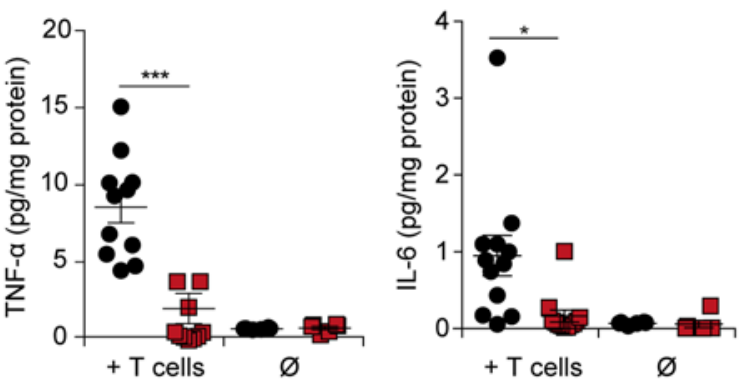

E
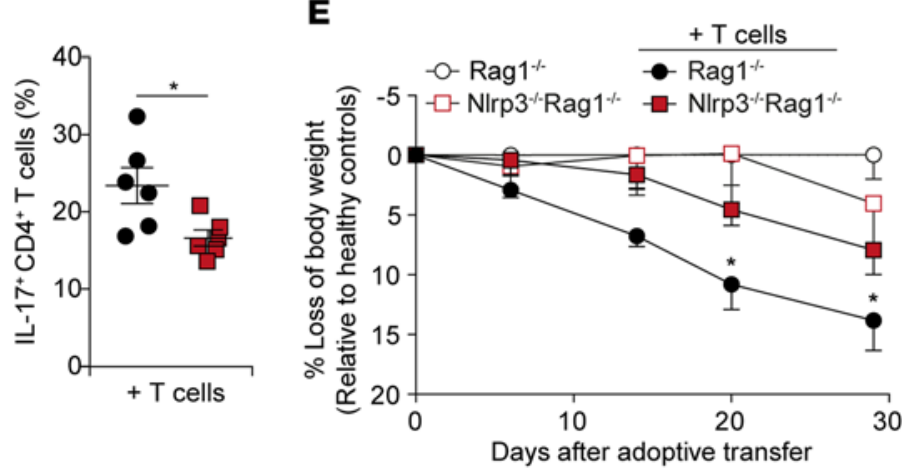

G

B
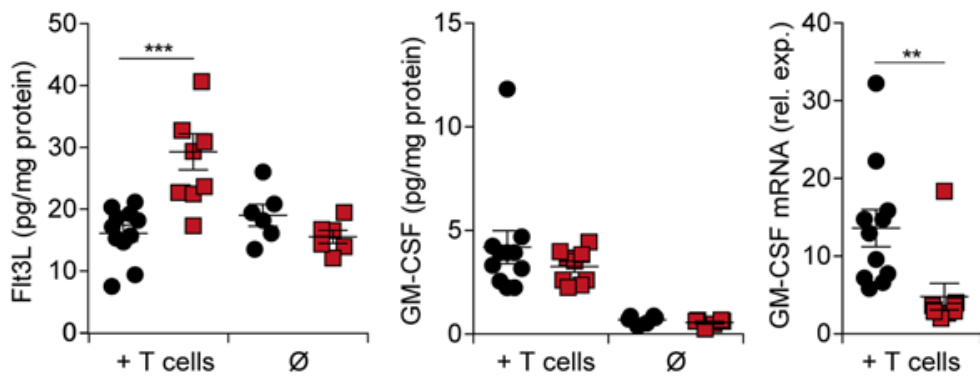

Days after adoptive transfer

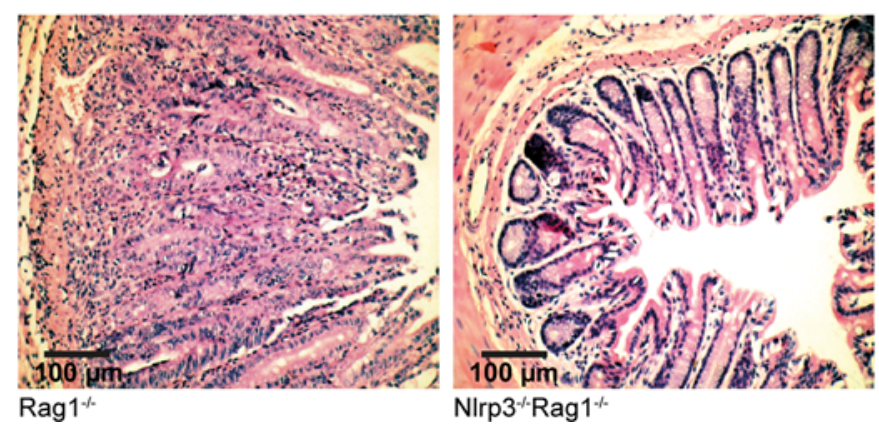

Figure 3. NLRP3 deficiency promotes $\mathrm{CD} 103^{+} \mathrm{DC}$ differentiation in a T cell transfer colitis model and protects mice from Th17-associated tissue

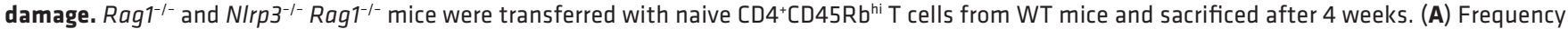
of total CD11 $C^{+}$DC and CD103+ DC in lamina propria, before and after T cell transfer, was analyzed by FACS. (B) Levels of FLT3L and GM-CSF in colon explants were analyzed by ELISA and qPCR. (C) Levels of proinflammatory cytokines IL-1 $\beta$, IL-18, TNF- $\alpha$, and IL-6 in colon explants were measured by ELISA. (D) Levels of IL-17 and IL-22 in colon and frequency of lamina propria IL-17+CD4+ $T$ cells were analyzed by ELISA and FACS, respectively. (E) Loss of body weight was monitored weekly and compared with appropriate healthy controls. (F) Clinical and histological scores at sacrifice. (C) H\&E staining of representative colon sections. Scale bars: $100 \mu \mathrm{m}$. Three independent experiments were performed. (A and B) Rag $1^{-/-}(+$transfer), $n=11$;

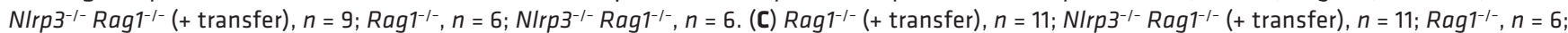

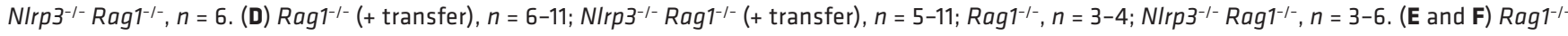

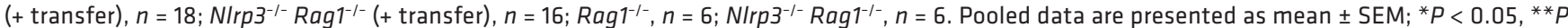
$<0.01,{ }^{* *} P<0.001$, as assessed by unpaired 2-tailed Student's $t$ test. For the body weight analysis, ${ }^{*} P<0.05$ according to 2 -way ANOVA followed by Sidak's multiple comparisons test. 
A

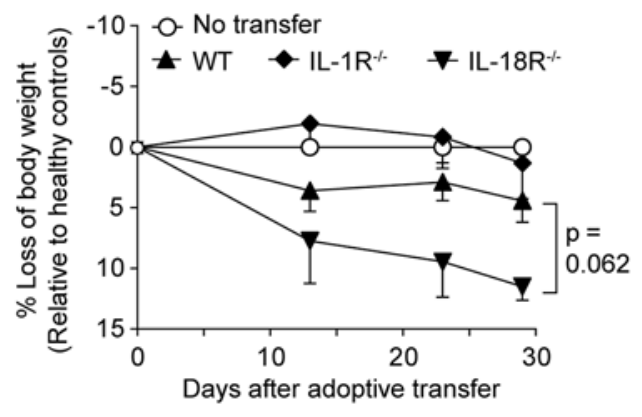

B

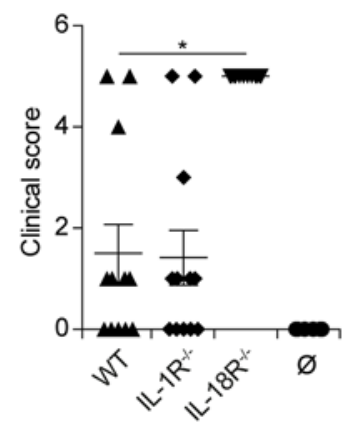

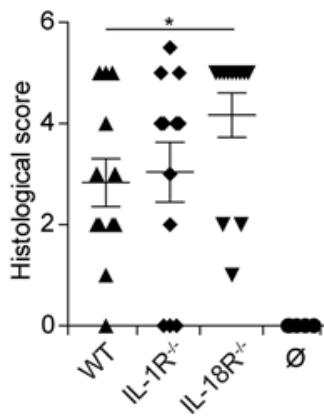

C

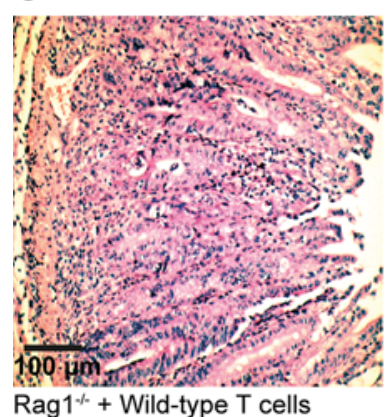

Rag $1^{1-}+$ Wild-type T cells

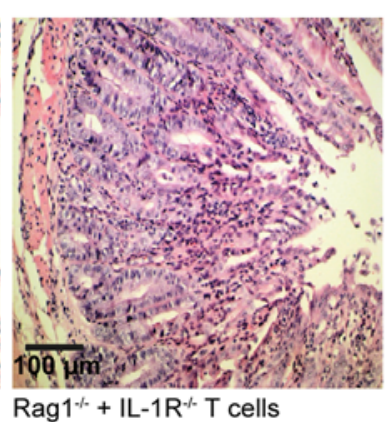

$\operatorname{Rag}^{1 /}+\mathrm{IL}-1 \mathrm{R}^{-/} \mathrm{T}$ cells

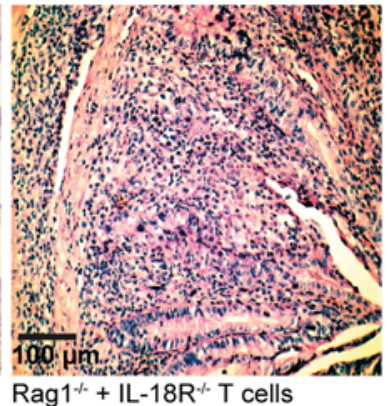

D

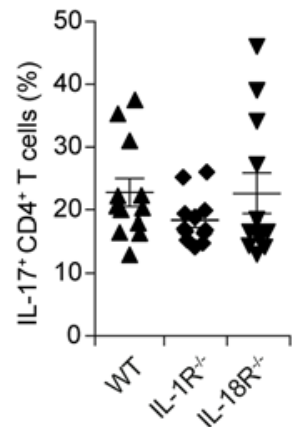

E
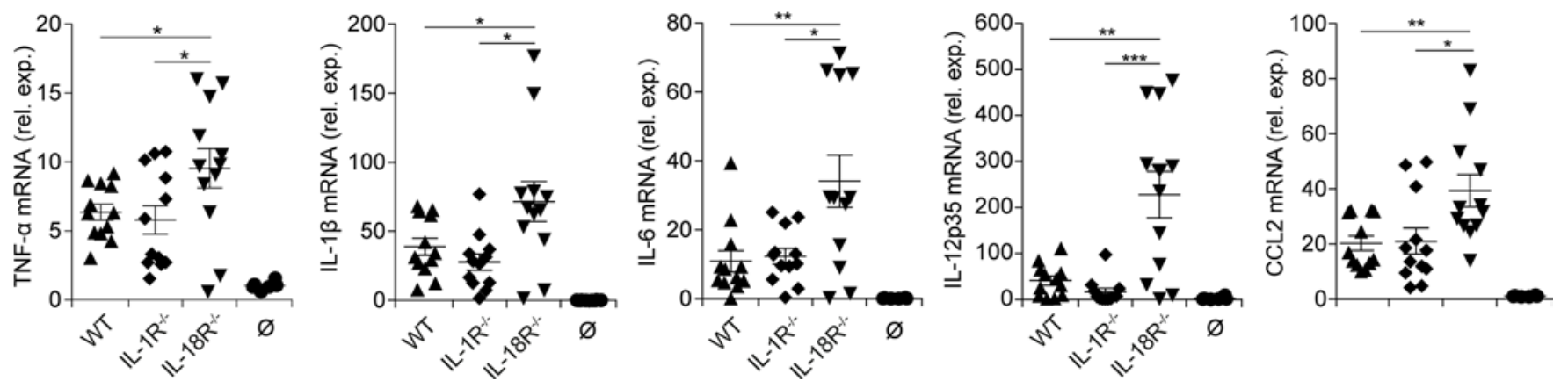

Figure 4. IL-18R deficiency in transferred CD4 ${ }^{+} T$ cells exacerbates colitis severity. Rag $1^{-1-}$ mice were transferred with naive CD4 $C D 45 R b^{\text {hi }} T$ cells from WT, IL-1R-deficient, or IL-18R-deficient mice and sacrificed after 4 weeks. (A) Loss of body weight over the course of 30 days after adoptive T cell transfer. (B) Clinical and histological scores at sacrifice. (C) H\&E staining of representative colon sections. Scale bars: $100 \mu \mathrm{m}$. (D) Frequency of total CD4+ T cells and IL-17+CD4+ $T$ cells isolated from lamina propria was analyzed with FACS. (E) Cytokine mRNA levels from colon explants were assessed by qPCR. Pooled data

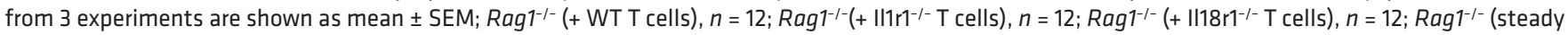
state), $n=6 .{ }^{*} P<0.05,{ }^{* *} P<0.01,{ }^{* *} P<0.001$, as assessed by unpaired 2-tailed Student's $t$ test.

induced more severe colitis with enhanced weight loss and severe destruction of the crypt architecture (Figure $4, \mathrm{~A}-\mathrm{C}$ ). Loss of body weight and histological analysis revealed similar pathology in WT- and $1 l 1 r 1^{-1-} \mathrm{T}$ cell-transferred mice within the first 28 days of colitis.

Higher disease activity in $I 118 \mathrm{rl}^{-1-} \mathrm{T}$ cell-transferred mice was paralleled by augmented $\mathrm{T}$ cell influx (Figure 4D) and increased colonic expression of proinflammatory cytokines and chemokines, such as TNF- $\alpha$, IL-1 $\beta$, IL-6, IL-12p35, and CCL2 (Figure 4E). These findings indicate a protective role for IL-18R signaling in T cells. The increase of NLRP3-dependent, myeloid cell-derived proinflammatory IL-1 $\beta$ shifts the balance of IL-1R and IL-18R T cell signaling from constitutively active IL-18R signaling toward proinflammatory IL-1R signaling.

Reduced intestinal inflammation in Nlrp3-deficient mice is preserved after cohousing. Genetic deletion of the inflammasome sensors Nlrp6 or Nlrc4 have been shown to result in microbial dysbiosis with blooming of bacterial taxa such as Prevotella or Salmonella spp $(23,41)$. To exclude a role for altered microbial 
A

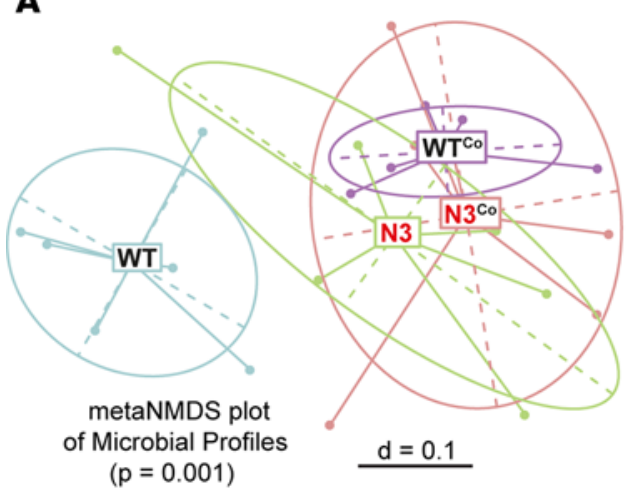

$(p=0.001)$
B

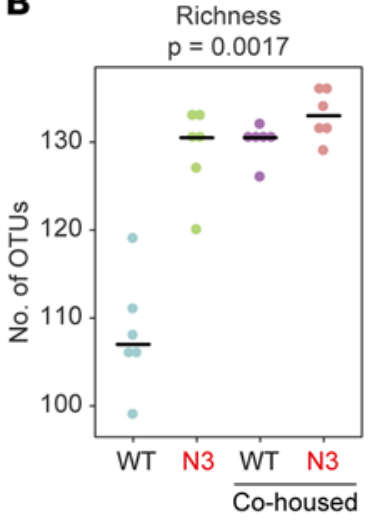

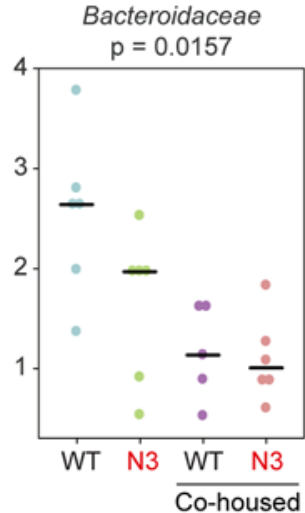
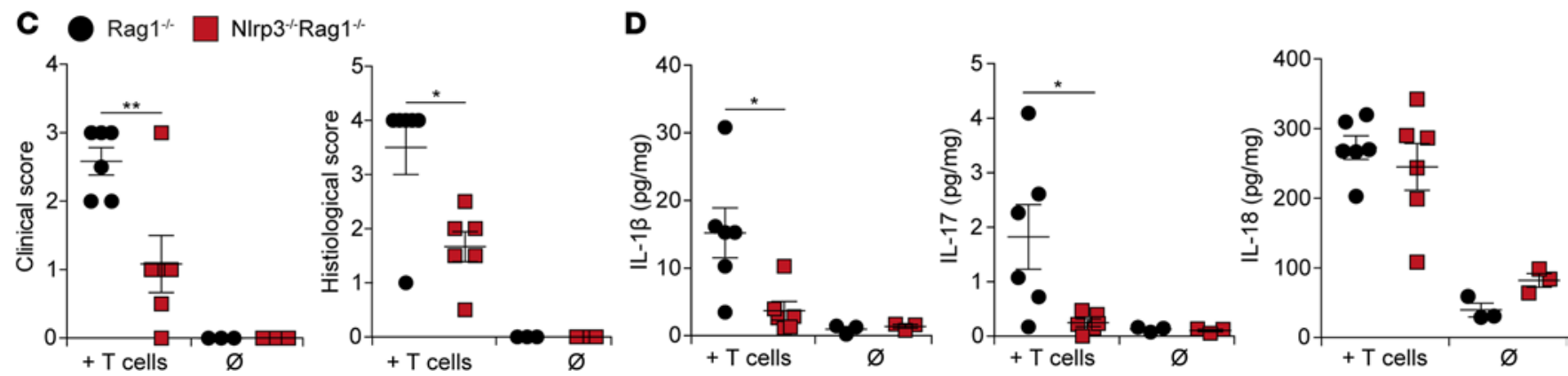

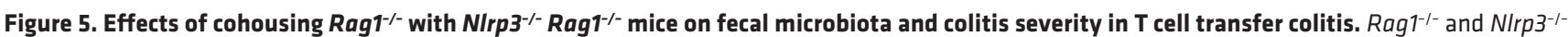
Rag1 $^{-1-}$ mice were cohoused for 3 weeks prior to transfer of naive CD4+CD45Rb hi T cells. (A) $\beta$-Diversity analysis of 165 rRNA gene amplicon-derived microbiota profiles. (B) Significant changes in $\alpha$ diversity and taxonomic groups. (C) Clinical and histological scores at sacrifice. (D) Cytokine secretion of

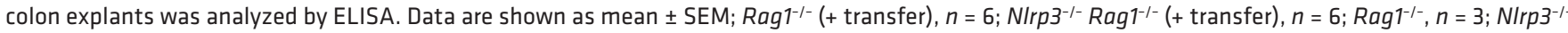
$\operatorname{Rag}^{1-1-}, n=3 ;{ }^{*} P<0.05,{ }^{*} P<0.01$, as assessed by unpaired 2-tailed Student's $t$ test.

composition between Rag1 $^{1_{-}^{--}}$and $\mathrm{Nlrp3}^{-/-}$Rag1 $^{1_{-}}$genotypes, mice were cohoused for 3 weeks prior to T cell transfer, and fecal microbiota was analyzed before and after cohousing by 16S rRNA gene amplicon sequencing. Multidimensional analysis of phylogenetic distances revealed that fecal microbiota diversity in $N$ lrp $3^{-/-} \mathrm{Rag}^{1^{-/}}$mice initially differed from that in $\mathrm{Rag}^{-/-}$mice. Within 3 weeks of cohousing, the phy-

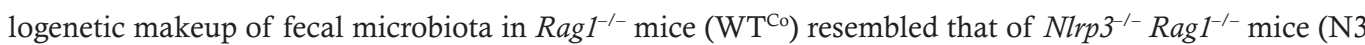
and $\mathrm{N}^{\mathrm{Co}}$ ) (Figure 5A). The changes in $\beta$ diversity after cohousing were accompanied by an increase in bacterial richness and in the relative abundance of sequences classified as Rikenellaceae (genus Alistipes), whereas proportions of Bacteroidaceae decreased (Figure 5B). At the level of single phylotypes, changes in the relative abundance of 8 unknown operational taxonomic units (OTUs) within the family S24-7 were observed (43), 6 decreased (nos. 2, 15, 16, 23, 33, 58) and 2 increased (nos. 3, 7), without significant relative abundance shifts at the level of the entire family (Supplemental Table 1; supplemental material available online with this article; https://doi.org/10.1172/jci.insight.96322DS1). After an additional 4 weeks of colitis induction under cohousing conditions, mice were sacrificed for clinical and histological

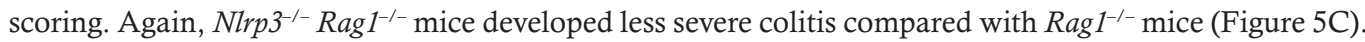
Similarly, colonic cytokine analysis showed a reduced Th17 immune profile, with lower levels of IL-1 $\beta$ and IL-17 and comparable IL-18 levels (Figure 5D). The data strongly argue for a canonical Nlrp3-based molecular mechanism in driving colitis pathology, irrespective of microbial colonization.

\section{Discussion}

We investigated the role of the Nlrp3 inflammasome in the differentiation of $\mathrm{CD} 103^{+} \mathrm{DC}$ and in intestinal inflammation. Using the $\mathrm{T}$ cell transfer colitis model, we show that NLRP3-mediated IL-1 $\beta$ release is the predominant factor responsible for the induction of Th17-mediated intestinal pathology. Reduced IL-1 $\beta$ levels in NLRP3-deficient mice were associated with accumulation of $\mathrm{CD} 103^{+} \mathrm{DC}$ and protection from colitis. Interestingly, IL-18 levels were identical in WT and $\mathrm{Nlrp}^{-/-}$mice, possibly involving other inflammasomes 


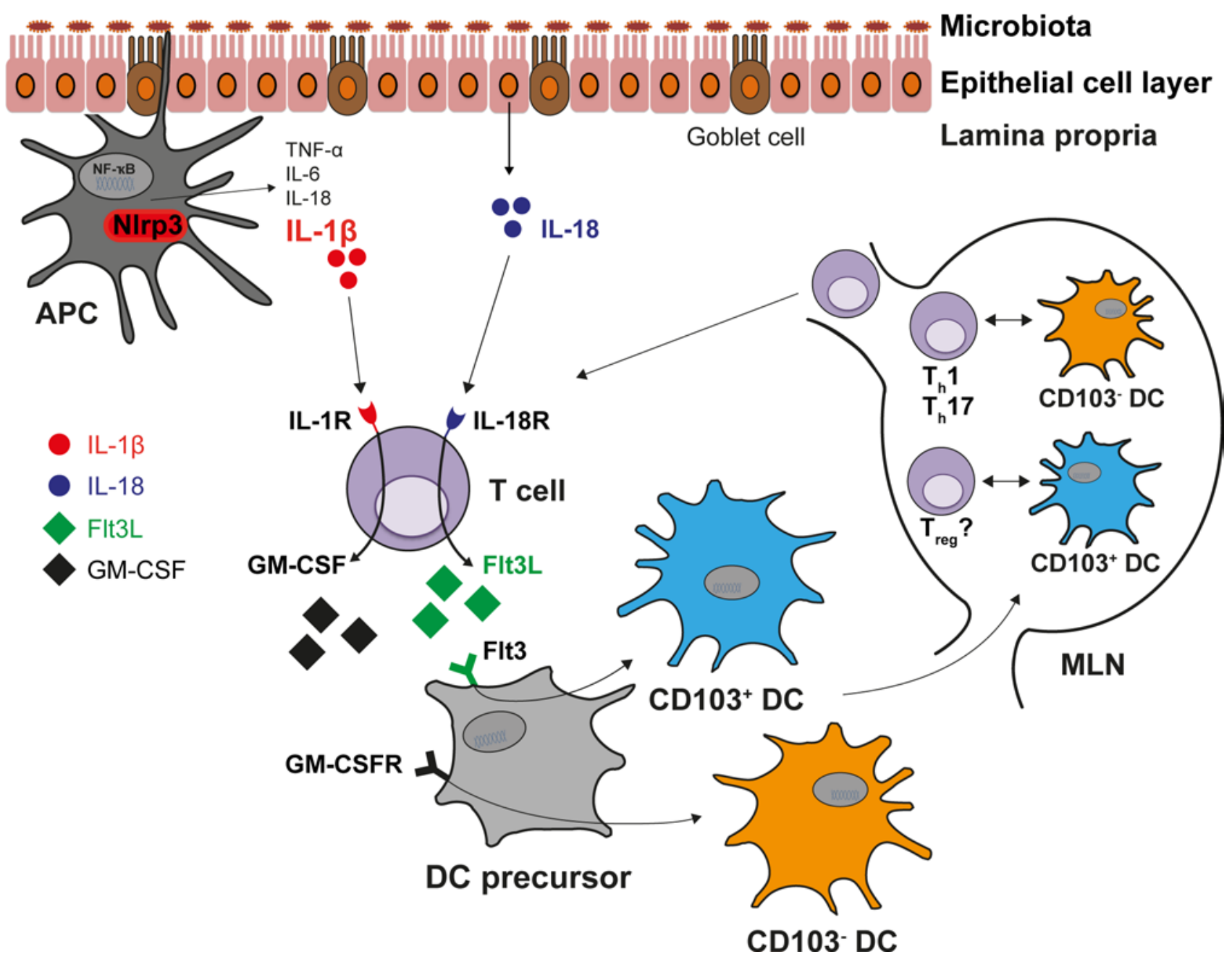

Figure 6. NLRP3-mediated IL-1 $\beta$ drives colitogenic Th17 immunity and regulates local DC phenotype by balancing GM-CSF and FLT3L production by T cells. Sensing of pathogenic patterns, by antigen-presenting cells (APC) in lamina propria (LP), results in enhanced IL-1 $\beta$ secretion in a NLRP3-dependent manner. Constitutive and NLRP3-independent IL-18 secretion by epithelial cells competes with IL-1 $\beta$ for T cell activation, regulating GM-CSF and FLT3L production by CD4 ${ }^{+}$T cells. In turn, the growth factor balance determines the differentiation of inflammatory CD103- DC and tolerogenic CD103+ DC from precursor cells, which polarize CD4+ $T$ cells in mesenteric lymph nodes either toward a Th1/Th17 or a tolerogenic phenotype, respectively.

$(18,23,42)$. Our data are concordant with a recent report on miR-223-mediated regulation of Nlrp3 expression and intestinal inflammation, describing Nlrp3-dependent elevation of IL-1, but not of IL-18, levels (20).

The activation of both IL-1 $\beta$ and IL-18 requires inflammasomes and caspase-1, with NLRP3 and NLRP6 as the best-characterized representatives. While NLRP3-dependent IL-1 $\beta$ is predominantly produced in immune cells, NLRP6 is highly expressed in intestinal epithelial cells and controls gut barrier integrity in an IL-18-dependent manner (18). Colonic IL-1 $\beta$ levels correlate with disease activity and are critical for Th17 differentiation (44). We found reduced IL-1 $\beta$ levels in NLRP3-deficient mice correlating with reduced Th17 cell infiltrates and tissue preservation. Conversely, IL-18 has been shown to signal via the IL-18R on T cells to limit Th17 cell differentiation (17). Accordingly, we show that transfer of IL-18Rdeficient $\mathrm{T}$ cells results in increased colitis pathology with vigorous $\mathrm{T}$ cell influx in the LP. The data argue that IL-18R signaling in T cells counteracts proinflammatory Nlrp3-dependent IL-1R signaling.

Contrary to published data on IL-1R deficiency of transferred $\mathrm{T}$ cells ameliorating colitis severity $(45,46)$, we did not observe significant differences between WT and $I l 1 r 1^{-1-} \mathrm{T}$ cells within the observation period of 4 weeks in our study, which focused on the early effects of inflammasome and cytokine signaling in colitis. This stands in contrast to the above-mentioned studies, which sacrificed the mice after 5-8 weeks, when mice were generally sicker. These findings hint toward a more prominent role of protective IL-18R signals in T cells in the early phase of colitis, whereas IL-1R-mediated effects occur later, when 
the epithelial barrier function is worsening and the LP is infiltrated with innate immune cells secreting IL-1 $\beta$. The precise kinetics of IL-18R and IL-1R signaling during $\mathrm{T}$ cell-mediated colitis remain to be elucidated in future studies.

Protection of NLRP3-deficient mice from colitis was associated with expansion of CD103 ${ }^{+}$DC. Their tolerogenic properties, potentially controlling disease severity, led us to investigate the DC/T cell axis. DC differentiation occurs via FLT3L and GM-CSF, which are produced by various immune and nonimmune cells upon inflammatory stimuli, including IL-1 family cytokines (47-50). We focused on IL-1 $\beta$ and IL-18, as inflammasome signaling pathways have already been shown to control cyto-

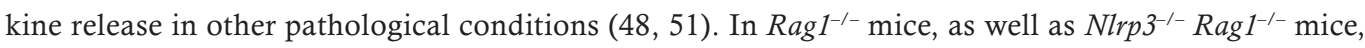
absence of T cells resulted in reduced DC numbers in steady state. T cells are a source of DC-expanding cytokines, such as FLT3L and GM-CSF, and have the potential to influence DC homeostasis in the gut $(51,52)$. We identified both IL-1 $\beta$ and IL-18 to control FLT3L and GM-CSF secretion by activated $\mathrm{T}$ cells in vitro and in vivo. Within this paradigm, IL-1 $\beta$ favored the secretion of GM-CSF, whereas IL-18 induced FLT3L release by activated T cells.

Both IL-1R and IL-18R signal through the adapter molecule MyD88 and share common downstream elements. Interestingly, IL-18 plays a role in IL-18R-mediated inhibition of IL-1R downstream signaling (17). The absence of NLRP3-mediated IL-1 $\beta$ may, thus, enforce IL-18R signaling, with enhanced FLT3L secretion and differentiation of $\mathrm{CD} 103^{+} \mathrm{DC}$. Conversely, IL-1R signaling enhances GM-CSF production by $\mathrm{T}$ cells, favoring inflammatory $\mathrm{CD} 103^{-} \mathrm{DC}$ to promote Th17 immunity (Figure 6).

IL-1 $\beta$ has been shown to promote Th17 responses in the intestine via the IL-1R, and mice as well as humans with hyperactive Nlrp3 mutations exhibit strong Th17 immunity as a consequence of excess IL-1 $\beta$ $(44,45,53)$. Accordingly, in our model, lack of NLRP3-dependent IL-1 $\beta$ was associated with decreased Th17 pathology with reduced levels of IL-1 $\beta$, IL-6, and IL-17. Finally, we identified IL-1 $\beta$ as potent stimulus for IL-22 secretion by T cells, which acts in concert with IL-17 to mount a robust Th17-based immune response. Impaired IL-22 secretion in $\mathrm{Nlrp}^{-/-}$mice further highlights the role for the Nlrp3 inflammasome in regulating Th17 immunity. However, IL-1 effects are not limited to Th17, as IL-1 signaling was shown to override Foxp3 expression, regulating plasticity of induced Tregs (54). The net effect of IL-1 $\beta$ is proinflammatory; however, the role of IL-18 is complex. Depending on site of activation, the release of IL-18 can mediate protective or destructive effects. IL-18 activation within the intestinal barrier contributes to the preservation of the intestinal barrier, while — in the LP — it mainly synergizes with IL-12, resulting in $\mathrm{T}$ cell activation. Recent data indicate that steady state intestinal epithelial cells constitutively secrete IL-18, which acts directly on IL-18R-expressing T cells, resulting in 2 effects: limitation of colonic Th17 differentiation and promotion of suppressive function of $\mathrm{FOXP}^{+} \mathrm{CD} 4^{+} \mathrm{T}$ cells, thereby preventing colitis (17). We suggest that IL-1 $\beta$ mediates increased susceptibility of NLRP3-sufficient mice toward T cell transfer colitis by overriding protective IL-18R signals, thereby shaping a highly plastic intestinal T cell pool. Dysbalance of IL-18R versus IL-1R signaling in T cells might explain beneficial versus detrimental effects of NLRP3-deficiency in the murine models of IBD, resulting in seemingly contradicting reports in the literature $(14,19)$.

The intestinal microbiota regulate gut homeostasis, and disrupted inflammasome signaling can result in dysbiosis with increased colonization of pathobionts $(23,29)$. Recently, Mamantopoulos et al. have questioned the role of inflammasomes in shaping intestinal ecology (55). In an attempt to control any potentially confounding microbiota effects, we cohoused mice and found that fecal microbiota profiles in WT mice shifted toward that in Nlrp3-deficient mice. Despite cohousing and acquisition of bacterial taxa in all cohoused mice, we observed the same protective phenotype in $N l r p 3^{-1-}$ mice, implying that the Nlrp3 inflammasome, not intestinal dysbiosis, modulates $\mathrm{T}$ cell responses and DC generation. This indicates that differences in intestinal ecology associated with NLRP3 deficiency are phenomena without causal influence on the outcome of $\mathrm{T}$ cell responses in transfer colitis. Our data might help to reconcile contradicting reports in the literature.

In summary, our data identify the NLRP3 inflammasome as an important checkpoint regulating the IL-1 $\beta /$ IL-18 ratio in the intestine to control immune homeostasis and Th17 immunity. The balance of IL-1 $\beta$ and IL-18 controls the secretion of FLT3L and GM-CSF by T cells, which in turn influences the differentiation of $\mathrm{CD}_{103}{ }^{+} \mathrm{DC}$ from their precursors. Pharmacological inhibition of the NNLRP3/IL-1 $\beta$ / GM-CSF axis might represent a promising approach for IBD treatment. 


\section{Methods}

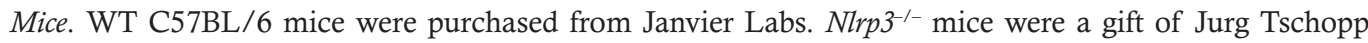

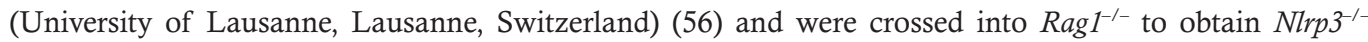
Rag $1^{-/-}$double-deficient mice. Rag $1^{-/-}$mice, originally obtained from The Jackson Laboratory, were a gift

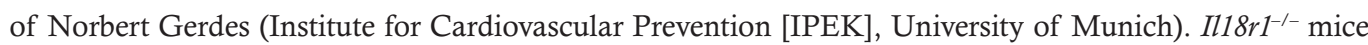

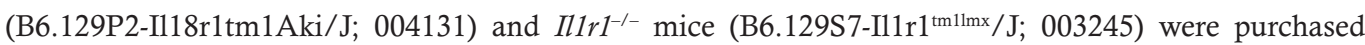
from The Jackson Laboratory. OT-II TCR transgenic mice (B6.Cg-Tg(TraTcrb)425Cbn $/ \mathrm{J}$ ) were a gift from Thomas Brocker (Institute of Immunology, University of Munich). Mice were kept on the C57BL/6 background for more than 15 generations and bred under SPF conditions with a 12-hour light/dark cycle, at the University of Munich. Mice were fed a standard chow diet (ssniff R/M-H autoclavable chow diet; ssniff Spezialdiäten), received water ad libitum, and were used for experiments between 8 and 10 weeks of age.

$T$ cell transfer colitis. Single cell suspensions from spleens of C57BL/6 mice were depleted of CD8 ${ }^{+}$, MHC class $\mathrm{II}^{+}, \mathrm{Mac}^{+}{ }^{+}$, and $\mathrm{B} 220^{+}$cells, leaving $\mathrm{CD}^{+} \mathrm{T}$ cells untouched (Dynabeads, Invitrogen). After staining with PE-conjugated CD4 and FITC-conjugated CD45Rb antibodies, naive $\mathrm{CD} 4^{+} \mathrm{CD} 45 \mathrm{Rb}^{\mathrm{hi}} \mathrm{T}$ cells were isolated using a BD FACSAria III cell sort system (>98\% purity). T cells $\left(4 \times 10^{5}\right)$ were adoptively transferred into $\mathrm{Rag}^{1^{-/}}$recipient mice via i.p. injection.

Clinical and histological scoring. Scoring of disease activity was done as previously described $(19,30)$. Briefly, combined diarrhea and hematochezia (clinical) scoring was done in a blinded fashion. Stool consistency was classified as: 0 , regular pellets; 1 , soft pellets; 2 , bulky pellets; 3 , pasty and opaque pellets; and 4 , liquid diarrhea. Hemoccult testing was staged as: 0 , negative; 2 , positive; 4 , visual rectal bleeding. The overall clinical score was calculated as the sum of the diarrhea and hematochezia scores, reaching a maximum of 8 points. Combined histological scoring was done for distal colon sections by a trained pathologist in a blinded fashion. Formalin-fixed distal colon samples $(1 \mathrm{~cm}$ each) were embedded in paraffin and cut into $4-\mu \mathrm{m}$ sections. Subsequently, H\&E staining was performed and analyzed. Focal inflammatory cell infiltration was graded as: 0, normal; 1, increased; 2, submucosal infiltration; and 3, transmural extension. Epithelial damage was scored as: 0 , no damage; 1 , scarce focal lesions; 2 , focal erosions or ulcerations of the mucosal layer; and 3 , severe damage extending to the intestinal wall. The overall histological score was calculated as the sum of the cell infiltration and epithelial damage scores, reaching a maximum of 6 points.

Ex vivo analysis of colonic cytokines. Colonic explants were weighed and cultured in complete RPMI media or shock frozen in liquid nitrogen, mechanically crushed, and incubated with cell lysis buffer (Bio$\mathrm{Rad}$ ) for 15 minutes on ice. The homogenate was centrifuged at $10,000 \mathrm{~g}$ at $4{ }^{\circ} \mathrm{C}$ for 15 minutes, and protein amounts were adjusted (Bradford analysis, Bio-Rad). Cytokines in supernatants or cell lysates were measured by ELISA for IL-6 (BD Biosciences); IL-22 (eBioscience); and GM-CSF, FLT3L, IL-1 $\beta$, TNF, IFN- $\gamma$, and IL-17 (all from R\&D Systems). Concentrations were normalized to tissue weight or whole protein, as indicated.

Analysis of LP immune cells. LP immune cells were isolated using Mouse Lamina Propria Dissociation Kit (Miltenyi Biotec). Colon sections were cut into small pieces and incubated with $5 \mathrm{mM}$ EDTA, $1 \mathrm{mM}$ DTT (MilliporeSigma), and 5\% FBS in HBSS buffer (Thermo Fisher Scientific) for 20 minutes and passed through a $70-\mu \mathrm{m}$ cell strainer. Remaining tissue was collected into a gentleMACS C tube (Miltenyi Biotec) containing 5\% FBS in HBSS (with $\mathrm{Ca}^{2+} / \mathrm{Mg}^{2+}$ ) and dissociated using gentleMACS Dissociator (Miltenyi Biotec). Cells were washed and stained for FACS analysis. For intracellular cytokine staining, cells were stimulated with $0.1 \mu \mathrm{g} / \mathrm{ml}$ PMA, $1 \mu \mathrm{g} / \mathrm{ml}$ ionomycin, $10 \mu \mathrm{g} / \mathrm{ml}$ Brefeldin A (all from MilliporeSigma) for 4 hours; washed; and stained for surface markers. Intracellular staining was done using BD Cytofix/Cytoperm solution (BD Biosciences).

Antibodies and flow cytometry. Rat anti-mouse CD4-PE (clone GK1.5), rat anti-mouse CD4-PerCP (clone RM4-5), rat anti-mouse CD45RB-FITC (clone 16A), rat anti-mouse IL-17 Alexa Fluor 488 (clone TC1118H10), and rat anti-mouse CD11b-PerCP-Cy5.5 (clone M1/70), as well as hamster anti-mouse CD3-APC (clone 145-2C11), were purchased from BD Biosciences. Hamster anti-mouse CD11c-Pacific Blue (clone N418), hamster anti-mouse CD103 Alexa Flour 488 (clone 2E7), hamster anti-mouse CD3-Pacific Blue (clone 145-2C11), and rat anti-mouse IFN- $\gamma$-Pacific Blue (clone XMG1.2) were from BioLegend. Flow cytometry was performed using a FACSCanto II system (BD Biosciences).

$q P C R$ analysis. Cells or colonic tissue were homogenized using an Ultra-turrax instrument (IKA). Total RNA was isolated with the peqGOLD total RNA kit (Peqlab) according to the manufacturers' protocol. Purity and yield were determined using NanoDrop (Thermo Fisher Scientific). cDNA transcription was done with the 
RevertAID First stranded cDNA Synthesis kit (Invitrogen), and qPCR analysis was performed with the Kapa Probe Fast Kit (Peqlab) on a LightCycler 480 system (Roche Diagnostics). $\beta$-Actin served as housekeeping gene.

Generation of BM-derived and splenic DC, and T cell coculture assay. BM cells of femur and tibia were passed through a $40-\mu \mathrm{m}$ cell strainer and centrifuged for 5 minutes at $400 \mathrm{~g}$. After RBC lysis (BD Biosciences), cells were incubated with recombinant murine IL-4 and GM-CSF $(20 \mathrm{ng} / \mathrm{ml})$, or FLT3L $(100 \mathrm{ng} / \mathrm{ml})$, for 7-9 days, and DC were isolated by magnetic beads (Miltenyi Biotec). If indicated, cells were stimulated with $0.5 \mu \mathrm{g} / \mathrm{ml}$ LPS (InvivoGen), and cytokine levels in supernatants were assessed. For coculture experiments, splenic DC were pulsed with or without $25 \mu \mathrm{g} / \mathrm{ml}$ MHC-II-restricted $\mathrm{OVA}_{323-339}$ peptide for 1 hour at $37^{\circ} \mathrm{C}$. Naive $\mathrm{CD}^{+}$ $\mathrm{T}$ cells were isolated by negative depletion (Miltenyi Biotec) from OT-II mice. DC $\left(2 \times 10^{4}\right)$ were cocultured with $1 \times 10^{5} \mathrm{CD}^{+} \mathrm{OT}$-II T cells for 5 days, and cytokine levels in supernatants were determined by ELISA.

$T$ cell stimulation assays. $\mathrm{CD}^{+} \mathrm{T}$ cells were isolated using $\mathrm{CD} 4^{+} \mathrm{T}$ Cell Isolation Kit mouse (Miltenyi Biotec) according to the manufacturer's instruction and were labeled with PerCP rat anti-mouse CD4 (clone RM4-5) and APC hamster anti-mouse CD3 (clone 145-2C11) (BD Biosciences) for FACS-sort (FACSAria III, BD Biosciences). Cells were resuspended in RPMI medium and activated with plate-bound anti-CD3 $(5 \mu \mathrm{g} / \mathrm{ml})$ and soluble anti-CD28 $(2 \mu \mathrm{g} / \mathrm{ml})$ in 96-well U-bottom plates for 72 hours. Where indicated, $20 \mathrm{ng} / \mathrm{ml}$ recombinant murine (rm) IL-1 $\beta$ (Peprotech) or $10 \mathrm{ng} / \mathrm{ml} \mathrm{rmIL-18} \mathrm{(BioVision)} \mathrm{was} \mathrm{added}$ to the cell cultures.

$16 S$ rRNA gene amplicon analysis. Fecal samples were collected before and 3 weeks after cohousing of animals, prior to $\mathrm{T}$ cell transfer. Samples were processed as described before (57). Briefly, genomic DNA was extracted using phenol/chloroform extraction after mechanical disruption. The variable region V3/V4 of $16 \mathrm{~S}$ rRNA genes was amplified using the primers $341 \mathrm{~F}$ and $785 \mathrm{R}$ following a 2-step procedure. Amplicons were purified using the AMPure XP system (Beckman Coulter) and sequenced in paired-end modus (PE275) using a MiSeq system (Illumina Inc.). Raw read files were processed using IMNGS (www.imngs. org) (43). OTUs were picked at a threshold of $97 \%$ sequence similarity. Only those with a relative abundance above $0.5 \%$ total sequences per sample were kept. Readouts and statistics were generated in $\mathrm{R}$ using Rhea (https://lagkouvardos.github.io/Rhea/) (43).

Statistics. GraphPad Prism 5 was used for data analysis. Two-tailed Student's $t$ test or 2-way ANOVA were used for group comparisons. Data are expressed as means \pm SEM, with at least 2 independent experiments performed. Differences were considered statistically significant at ${ }^{*} P<0.05,{ }^{* *} P<0.01,{ }^{* *} P<0.001$.

Study approval. Animal experiments were reviewed by an animal care authority and approved by the Regierung von Oberbayern.

\section{Author contributions}

$\mathrm{PD}, \mathrm{CB}$, and $\mathrm{MS}$ conceived the experimental strategy. $\mathrm{PD}, \mathrm{RM}, \mathrm{CH}, \mathrm{CR}$, and $\mathrm{CB}$ designed and performed experiments. HAL contributed histological analysis. GD and SH performed experiments on $I l 18 r 1^{-/-}$mice. TC and SF contributed the microbiota analysis. SK and SE gave conceptual advice. The paper was written by $\mathrm{PD}, \mathrm{CB}$, and MS.

\section{Acknowledgments}

We thank Michael Hristov (IPEK, Ludwig-Maximilians-Universität [LMU] Munich) for assistance with cell sorting; Nicole Lichter and Cäcillia Czogalla for technical assistance in animal work and with ex vivo assays; Caroline Ziegler and Angela Sachsenhauser for technical assistance with sample preparation for high-throughput 16S rRNA gene amplicon analysis; Irmina Karl for technical assistance; and Thomas Korn (Neuro-Kopf-Zentrum, Technical University of Munich, Munich, Germany) and Norbert Gerdes (IPEK,

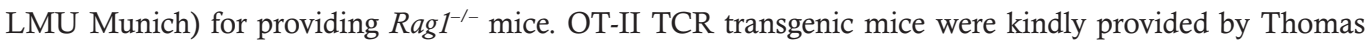
Brocker (Institute of Immunology, University of Munich). Flt3L-transduced B16 melanoma cells were provided by Glenn Dranoff (Dana-Farber Cancer Institute, Boston, Massachusetts, USA). This work was funded by Else Kröner-Fresenius-Stiftung 2012_A27 to CB and Deutsche Forschungs-gemeinschaft - SFB1123 to PD and MS.

Address correspondence to: Max Schnurr, Klinikum der Universität München, Lindwurmstrasse 2a, Munich 80337, Germany. Phone: 49.89.4400.57322; Email: max.schnurr@med.uni-muenchen.de. 
1. de Souza HS, Fiocchi C. Immunopathogenesis of IBD: current state of the art. Nat Rev Gastroenterol Hepatol. 2016;13(1):13-27.

2. Neurath MF. Cytokines in inflammatory bowel disease. Nat Rev Immunol. 2014;14(5):329-342.

3. Hugot JP, et al. Association of NOD2 leucine-rich repeat variants with susceptibility to Crohn's disease. Nature. 2001;411(6837):599-603.

4. Ogura Y, et al. A frameshift mutation in NOD2 associated with susceptibility to Crohn's disease. Nature. 2001;411(6837):603606.

5. Martinon F, Burns K, Tschopp J. The inflammasome: a molecular platform triggering activation of inflammatory caspases and processing of proIL-beta. Mol Cell. 2002;10(2):417-426.

6. Latz E, Xiao TS, Stutz A. Activation and regulation of the inflammasomes. Nat Rev Immunol. 2013;13(6):397-411.

7. Paramel GV, Sirsjö A, Fransén K. Role of genetic alterations in the NLRP3 and CARD8 genes in health and disease. Mediators Inflamm. 2015;2015:846782.

8. Casini-Raggi V, Kam L, Chong YJ, Fiocchi C, Pizarro TT, Cominelli F. Mucosal imbalance of IL-1 and IL-1 receptor antagonist in inflammatory bowel disease. A novel mechanism of chronic intestinal inflammation. J Immunol. 1995;154(5):2434-2440.

9. Pizarro TT, et al. IL-18, a novel immunoregulatory cytokine, is up-regulated in Crohn's disease: expression and localization in intestinal mucosal cells. J Immunol. 1999;162(11):6829-6835.

10. Cominelli F, et al. Interleukin 1 (IL-1) gene expression, synthesis, and effect of specific IL-1 receptor blockade in rabbit immune complex colitis. J Clin Invest. 1990;86(3):972-980.

11. Reinecker HC, et al. Enhanced secretion of tumour necrosis factor-alpha, IL-6, and IL-1 beta by isolated lamina propria mononuclear cells from patients with ulcerative colitis and Crohn's disease. Clin Exp Immunol. 1993;94(1):174-181.

12. Ludwiczek $\mathrm{O}$, et al. Imbalance between interleukin-1 agonists and antagonists: relationship to severity of inflammatory bowel disease. Clin Exp Immunol. 2004;138(2):323-329.

13. Raza A, Yousaf W, Giannella R, Shata MT. Th17 cells: interactions with predisposing factors in the immunopathogenesis of inflammatory bowel disease. Expert Rev Clin Immunol. 2012;8(2):161-168.

14. Zaki MH, Boyd KL, Vogel P, Kastan MB, Lamkanfi M, Kanneganti TD. The NLRP3 inflammasome protects against loss of epithelial integrity and mortality during experimental colitis. Immunity. 2010;32(3):379-391.

15. Siegmund B. Interleukin-18 in intestinal inflammation: friend and foe? Immunity. 2010;32(3):300-302

16. Reuter BK, Pizarro TT. Commentary: the role of the IL-18 system and other members of the IL-1R/TLR superfamily in innate mucosal immunity and the pathogenesis of inflammatory bowel disease: friend or foe? Eur J Immunol. 2004;34(9):2347-2355.

17. Harrison OJ, et al. Epithelial-derived IL-18 regulates Th17 cell differentiation and Foxp3 $3^{+}$Treg cell function in the intestine. Mucosal Immunol. 2015;8(6):1226-1236.

18. Nowarski R, et al. Epithelial IL-18 Equilibrium Controls Barrier Function in Colitis. Cell. 2015;163(6):1444-1456.

19. Bauer C, et al. Colitis induced in mice with dextran sulfate sodium (DSS) is mediated by the NLRP3 inflammasome. Gut. 2010;59(9):1192-1199.

20. Neudecker V, et al. Myeloid-derived miR-223 regulates intestinal inflammation via repression of the NLRP3 inflammasome. J Exp Med. 2017;214(6):1737-1752.

21. Siegmund B, Lehr HA, Fantuzzi G, Dinarello CA. IL-1 beta -converting enzyme (caspase-1) in intestinal inflammation. Proc Natl Acad Sci USA. 2001;98(23):13249-13254.

22. Bauer C, et al. The ICE inhibitor pralnacasan prevents DSS-induced colitis in C57BL/6 mice and suppresses IP-10 mRNA but not TNF-alpha mRNA expression. Dig Dis Sci. 2007;52(7):1642-1652.

23. Elinav E, et al. NLRP6 inflammasome regulates colonic microbial ecology and risk for colitis. Cell. 2011;145(5):745-757.

24. Allen IC, et al. The NLRP3 inflammasome functions as a negative regulator of tumorigenesis during colitis-associated cancer. J Exp Med. 2010;207(5):1045-1056.

25. Takagi H, et al. Contrasting action of IL-12 and IL-18 in the development of dextran sodium sulphate colitis in mice. Scand $J$ Gastroenterol. 2003;38(8):837-844.

26. Siegmund B, et al. Neutralization of interleukin-18 reduces severity in murine colitis and intestinal IFN-gamma and TNF-alpha production. Am J Physiol Regul Integr Comp Physiol. 2001;281(4):R1264-R1273.

27. Sivakumar PV, et al. Interleukin 18 is a primary mediator of the inflammation associated with dextran sulphate sodium induced colitis: blocking interleukin 18 attenuates intestinal damage. Gut. 2002;50(6):812-820.

28. Holmkvist P, Pool L, Hägerbrand K, Agace WW, Rivollier A. IL-18R $\alpha$-deficient CD4(+) T cells induce intestinal inflammation in the CD45RB(hi) transfer model of colitis despite impaired innate responsiveness. Eur J Immunol. 2016;46(6):1371-1382.

29. Hirota SA, et al. NLRP3 inflammasome plays a key role in the regulation of intestinal homeostasis. Inflamm Bowel Dis. 2011;17(6):1359-1372

30. Bauer C, Duewell P, Lehr HA, Endres S, Schnurr M. Protective and aggravating effects of Nlrp3 inflammasome activation in IBD models: influence of genetic and environmental factors. Dig Dis. 2012;30 Suppl 1:82-90.

31. Cerovic V, et al. Intestinal CD103(-) dendritic cells migrate in lymph and prime effector T cells. Mucosal Immunol. 2013;6(1):104-113.

32. Coombes JL, et al. A functionally specialized population of mucosal CD103+ DCs induces Foxp3+ regulatory T cells via a TGF-beta and retinoic acid-dependent mechanism. J Exp Med. 2007;204(8):1757-1764.

33. Laffont S, Siddiqui KR, Powrie F. Intestinal inflammation abrogates the tolerogenic properties of MLN CD103+ dendritic cells Eur J Immunol. 2010;40(7):1877-1883.

34. Uematsu S, et al. Regulation of humoral and cellular gut immunity by lamina propria dendritic cells expressing Toll-like receptor 5. Nat Immunol. 2008;9(7):769-776.

35. Niess JH, et al. CX3CR1-mediated dendritic cell access to the intestinal lumen and bacterial clearance. Science. 2005;307(5707):254-258.

36. Pabst O, Bernhardt G. The puzzle of intestinal lamina propria dendritic cells and macrophages. Eur J Immunol. 2010;40(8):2107-2111

37. Bogunovic M, et al. Origin of the lamina propria dendritic cell network. Immunity. 2009;31(3):513-525.

38. Varol C, et al. Intestinal lamina propria dendritic cell subsets have different origin and functions. Immunity. 2009;31(3):502-512

39. Miller G, Pillarisetty VG, Shah AB, Lahrs S, DeMatteo RP. Murine Flt3 ligand expands distinct dendritic cells with both tolero- 
genic and immunogenic properties. J Immunol. 2003;170(7):3554-3564.

40. Sims JE, Smith DE. The IL-1 family: regulators of immunity. Nat Rev Immunol. 2010;10(2):89-102.

41. Franchi L, et al. NLRC4-driven production of IL-1 $\beta$ discriminates between pathogenic and commensal bacteria and promotes host intestinal defense. Nat Immunol. 2012;13(5):449-456.

42. Wilson NS, et al. Inflammasome-dependent and -independent IL-18 production mediates immunity to the ISCOMATRIX adjuvant. J Immunol. 2014;192(7):3259-3268.

43. Lagkouvardos I, et al. The Mouse Intestinal Bacterial Collection ( $\mathrm{miBC}$ ) provides host-specific insight into cultured diversity and functional potential of the gut microbiota. Nat Microbiol. 2016;1(10):16131.

44. Lasigliè D, et al. Role of IL-1 beta in the development of human T(H)17 cells: lesson from NLPR3 mutated patients. PLoS One. 2011;6(5):e20014.

45. Coccia M, et al. IL-1 $\beta$ mediates chronic intestinal inflammation by promoting the accumulation of IL-17A secreting innate lymphoid cells and CD4(+) Th17 cells. J Exp Med. 2012;209(9):1595-1609.

46. Li B, Gurung P, Malireddi RK, Vogel P, Kanneganti TD, Geiger TL. IL-10 engages macrophages to shift Th17 cytokine dependency and pathogenicity during T-cell-mediated colitis. Nat Commun. 2015;6:6131

47. Brasel K, Escobar S, Anderberg R, de Vries P, Gruss HJ, Lyman SD. Expression of the flt3 receptor and its ligand on hematopoietic cells. Leukemia. 1995;9(7):1212-1218.

48. Guermonprez P, et al. Inflammatory Flt31 is essential to mobilize dendritic cells and for T cell responses during Plasmodium infection. Nat Med. 2013;19(6):730-738.

49. Huleihel M, Douvdevani A, Segal S, Apte RN. Different regulatory levels are involved in the generation of hemopoietic cytokines (CSFs and IL-6) in fibroblasts stimulated by inflammatory products. Cytokine. 1993;5(1):47-56.

50. Solanilla A, et al. Expression of Flt3-ligand by the endothelial cell. Leukemia. 2000;14(1):153-162.

51. Lukens JR, Barr MJ, Chaplin DD, Chi H, Kanneganti TD. Inflammasome-derived IL-1 $\beta$ regulates the production of GM-CSF by CD4(+) T cells and $\gamma \delta$ T cells. J Immunol. 2012;188(7):3107-3115.

52. Saito Y, Boddupalli CS, Borsotti C, Manz MG. Dendritic cell homeostasis is maintained by nonhematopoietic and T-cell-produced Flt3-ligand in steady state and during immune responses. Eur J Immunol. 2013;43(6):1651-1658.

53. Meng G, Zhang F, Fuss I, Kitani A, Strober W. A mutation in the Nlrp3 gene causing inflammasome hyperactivation potentiates Th17 cell-dominant immune responses. Immunity. 2009;30(6):860-874.

54. Basu R, et al. IL-1 signaling modulates activation of STAT transcription factors to antagonize retinoic acid signaling and contro the TH17 cell-iTreg cell balance. Nat Immunol. 2015;16(3):286-295.

55. Mamantopoulos M, et al. Nlrp6- and ASC-Dependent Inflammasomes Do Not Shape the Commensal Gut Microbiota Composition. Immunity. 2017;47(2):339-348.e4.

56. Martinon F, Pétrilli V, Mayor A, Tardivel A, Tschopp J. Gout-associated uric acid crystals activate the NALP3 inflammasome. Nature. 2006;440(7081):237-241

57. Lagkouvardos I, et al. Gut metabolites and bacterial community networks during a pilot intervention study with flaxseeds in healthy adult men. Mol Nutr Food Res. 2015;59(8):1614-1628. 